\title{
Behavioral assessment of emotion discrimination, emotion regulation, and cognitive control in childhood, adolescence, and adulthood
}

\author{
Nim Tottenham ${ }^{1,2 *}$, Todd A. Hare ${ }^{3}$ and B. J. Casey ${ }^{2}$ \\ Developmental Affective Neuroscience Laboratory, Department of Psychology, University of California at Los Angeles, Los Angeles, CA, USA \\ 2 Sackler Institute for Developmental Psychobiology, Weill Cornell Medical College, New York, NY, USA \\ ${ }^{3}$ Neuroeconomics Laboratory, Division of Humanities and Social Sciences, California Institute of Technology, Pasadena, CA, USA
}

Edited by:

Susan M. Rivera, University of

California, USA

Reviewed by:

Kristin Hansen Lagattuta, University of

California at Davis, USA

Amanda Guyer, University of California, USA

\section{*Correspondence:}

Nim Tottenham, Department of

Psychology, University of California at

Los Angeles, 2243C Franz Hall, Los

Angeles, CA 90095, USA.

e-mail:nimtottenham@ucla.edu
Emotion discrimination, emotion regulation, and cognitive control are three related, yet separable processes that emerge over the course of development. The current study tested 100 children, adolescents, and adults on an Emotional Go/Nogo task, illustrating the ability of this paradigm to identify the unique developmental patterns for each of these three processes in the context of both positive (happy) and negative emotions (fear, sad, and anger), across three different age groups. Consistent with previous literature, our findings show that emotion discrimination and regulatory abilities (both cognitive control and emotion regulation) improve steadily for each age group, with each age group showing unique patterns of performance. The findings suggest that emotion regulation is constructed from basic cognition control and emotion discrimination skills. The patterns of behavior from the Emotional Go/Nogo task provide normative benchmark data across a wide range of emotions that can be used for future behavioral and neuroimaging studies that examine the developmental construction of emotion regulatory processes.

\section{INTRODUCTION}

Cognitive control is a heterogeneous construct that has been defined as the ability to actively maintain representations of goals and the means to achieve them (Duncan, 2001; Miller and Cohen, 2001). One well-studied aspect of cognitive control is the ability to withhold a prepotent behavioral response, an ability that undergoes a protracted developmental course (reviewed in Casey et al., 2002). When cognitive control processes interact with emotional information, as commonly occurs in daily life, a second regulatory process is engaged, often labeled emotion regulation, a single term that encompasses several behavioral constructs, that collectively describe systematic changes that occur when emotions are activated (Cole et al., 2004). These changes include two types of regulatory phenomena, those processes that regulate an emotion (Gross, 1998) and those processes (e.g., memory, behavior) that are regulated by emotions (Campos et al., 2004; Cole et al., 2004). While much work has focused on the former (reviewed in Ochsner and Gross, 2005), the present study focused on the development of the latter aspect of emotion regulation, which is the ability to maintain cognitive control in the context of potentially interfering emotional information. The objectives of this study are to: (1) characterize age-related differences in cognitive control in the absence of and in the presence of emotional information (emotion regulation); and (2) provide a standard against which emotion regulation related processes can be measured in behavioral, clinical, and neuroimaging studies across development.

Regulating one's behavior is particularly difficult early in life even in the absence of emotional information, and young children are notoriously poor at behavioral regulation (Mischel and
Underwood, 1974). Previous work has shown that the development of cognitive control extends into late adolescence and early adulthood (Casey et al., 1997; Durston et al., 2002). Evidence for developmental change in cognitive control is based on numerous and varied laboratory tasks. Cognitive control has been measured in infants with the A, not B task (Piaget, 1954; Diamond, 1985), in toddlers with laboratory motor and patience tasks (Kochanska et al., 2000), in preschool-aged children with card sorting tasks (Zelazo et al., 1996; Munakata and Yerys, 2001), and school-aged children with Stroop-like tasks (Tipper et al., 1989; Marsh et al., 2006) and computerized tasks like flanker (Bunge et al., 2002), stop-signal (Oosterlaan et al., 1998), and go/nogo tasks (Casey et al., 1997). These studies have all shown that cognitive control processes are poor at younger ages, but because of potential ceiling effects in adult samples (Lagattuta et al., in press), it is most useful to employ tasks that can be used across multiple age groups. Traditional go/nogo paradigms, the type of task used in the current study, have been used across multiple age groups and show that children make more false alarm errors (i.e., demonstrate weaker cognitive control) relative to adults. The go/nogo task shows reliable and robust behavioral effects across multiple age groups (Casey et al., 1997; Durston et al., $2002,2006)$ making it an attractive neuropsychological tool, and in this paper we have modified the traditional go/nogo task to examine cognitive control when emotional information is present and when it is absent.

The frequency of emotional stimulation in daily life motivated the examination of cognitive control in the context of emotional information and how this aspect of emotion regulation changes with age. Facial expressions are a rich source of emotional information, and evidence from infant research suggests that the ability to 
discriminate basic emotions emerges quite early, within the first year of life (Nelson and Dolgin, 1985; Flom and Bahrick, 2007), although this ability continues to develop throughout childhood and adolescence (Lenti et al., 1999; Thomas et al., 2007). Previous work has shown age-related changes in basic emotion discrimination tasks (McClure, 2000; Herba and Phillips, 2004; Herba et al., 2006; Thomas et al., 2007), where age-related improvements continue to be observed through early adulthood. The current study builds on existing literature by testing very rapid emotion discrimination in the context of a go/nogo task in order to examine how discriminating between facial expressions of emotion interacts with control processes. Because of the ability of facial expressions to bias behavioral performance, here we examined age-related differences in exerting cognitive control in the presence and absence of emotional facial expressions. Previous studies have examined emotion regulation in various age groups, using separate, age-appropriate paradigms, for example: delay of gratification in preschoolers (Mischel and Underwood, 1974), Stroop task reaction times for children (Kindt et al., 1997), and simulated driving performance for adolescents (Steinberg et al., 2008). In the current study, we used a single task that is appropriate for child, adolescent, and adult subjects to characterize age-group related changes in emotion regulation. The use of a single task for all age groups allows for direct comparisons of behavioral performance between age groups.

Emotion researchers have outlined the empirical challenges in assessing emotion regulation, which include creating a task (a) that is appropriate for both young children and adults (Eisenberg et al., 2004) and (b) creating a task that allows for a comparison of emotion and regulatory phenomena in contrasting conditions (Cole et al., 2004). In the current study, we attempted to address these challenges by using an Emotional Go/Nogo task, that: (1) can be administered across a broad range of ages (in this study we tested 5- to 28-year olds); and (2) can be used to examine regulation under multiple emotional conditions, including positive, negative, and neutral facial expressions. This task is appropriate for young children as well as challenging for adults. Moreover, rather than passively viewing emotions or attending to non-emotional features of the stimuli, this task directly measures the ability to approach or avoid the relevant emotional information and has been validated in neuroimaging studies to dissociate top down prefrontal cognitive systems from subcortical limbic regions for both negative and positive emotions (Hare et al., 2008; Somerville et al., in press). We tested whether it is possible to identify developmental periods when behavioral patterns associated with cognitive control, emotion regulation, and facial expression discrimination emerge and/or demonstrate rapid age-related change. Although the literature has previously addressed these processes separately, here we simultaneously examined behavioral performance of these three typically co-occurring processes for direct comparison and to provide normative data on a neuropsychological tool that can be used across a wide age range. We tested the hypotheses that in a healthy sample of children, adolescents, and adults (1) emotional information can interfere with cognitive control processes, and (2) there will be unique age-group related improvements in these three skills.

\section{MATERIALS AND METHODS SUBJECTS}

We recruited 101 subjects and present data from a sample of 100 healthy volunteers (51 males and 49 females). These subjects included 53 children [27 females; 5-12 years old; mean $(S D)=8.64(1.95)], 24$ adolescents $[12$ females; $13-18$ years old; mean $(\mathrm{SD})=15.25(1.56)$ ], and 23 adults [ 10 females; $19-28$ years old; mean $(\mathrm{SD})=23.74(2.05)]$. The remaining subject was excluded because of incomplete data. Subjects were included if they had no history of neurologic or psychiatric disorder (as measured by telephone screening) and had an estimated full scale IQ of greater than 80 as measured by the Wechsler Abbreviated Scale of Intelligence (WASI; Wechsler, 1999). All subjects or their parents (if minors) gave both verbal and written consent before the task was administered.

\section{STIMULI}

The set of stimuli consisted of grayscale images of 10 adults (five males and five females; Ekman and Friesen, 1976) posing five different expressions (happy, fearful, angry, sad, and neutral). Visual angle was approximately $12^{\circ}$.

\section{BEHAVIORAL PARADIGM}

The task required participants to press a button when a given facial expression target (e.g., neutral) was displayed. Face stimuli were presented singly in the center of the screen (Figure 1). Subjects were instructed to press a button as fast as they could when a named expression was presented. These "go" trials occurred frequently (70\% of the trials) in order to create a prepotent tendency for the subject to respond. Participants were instructed to withhold pressing a button for a "nogo" facial expression stimulus (e.g., fearful face), and these "nogo" trials occurred infrequently (30\% of the trials). Subjects were not told what the "nogo" faces were, but were instructed to withhold pressing for "any face other than the 'go' expression.” In each block, an emotional expression (happy, fear, angry, or sad) was always paired with a neutral expression, and depending on the block, either the emotional expression served as the "go" stimulus (when neutral was the "nogo" stimulus) or as the "nogo" stimulus (when neutral was the "go" stimulus). Therefore, eight randomized blocks of "go-nogo" pairs (happy-neutral,

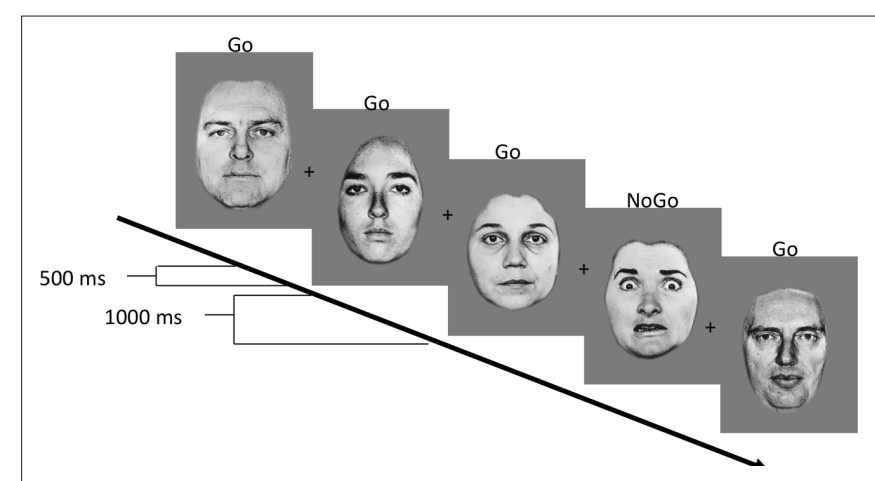

FIGURE 1 | Illustration of task. In this example, neutral faces are the "go" stimulus, and fear faces are the "nogo" stimulus. 
neutral-happy, fear-neutral, neutral-fear, angry-neutral, neutralangry, sad-neutral, and neutral-sad) with 30 randomized trials for each condition were administered to participants. Stimulus duration was $500 \mathrm{~ms}$ with $1000 \mathrm{~ms}$ between trials to ensure that participants had enough time to respond. Practice trials were administered to ensure that participants understood the task and could execute the responses. Stimulus presentation and response collection was programmed using Psyscope software (Cohen et al., 1993) and run on an Apple computer.

\section{DATA ANALYSIS PLAN}

There were four levels of the emotion condition for each analysis, which were happy, fear, angry, and sad emotional expressions. Within each block, these emotions were always paired with a neutral face for each condition such that if an emotional expression was the "go" target, then a neutral facial expression was the "nogo" non-target. Likewise, if an emotional face was the "nogo" non-target then a neutral face was the "go" target within that block of trials.

To fully capture the age-group related changes in behavior, we calculated the measures d-prime, false alarm rate, miss rate, reaction time, and the speed/accuracy trade-off for each age group. We operationally defined the d-prime measure as our primary index of emotion recognition. D-prime, which provides an index of accuracy accounting for response bias, was calculated by subtracting the $\mathrm{z}$-transformed false alarm rate from the $\mathrm{z}$-transformed hit rate. Overall false alarm rate (commission errors) was our index of cognitive control, and false alarm rate specifically to emotional "nogo" stimuli was our index of emotion regulation. False alarm rate and miss rate were calculated as the proportion of total possible errors for each type, and false alarm rate was used in our calculation of speed/accuracy trade-offs by age group. Because this task also requires an additional aspect of emotion regulation, which is the ability to approach a negatively valenced stimulus (e.g., angry faces), we examined reaction time differences to these expressions across the three age groups. Reaction times for hits were calculated for correct trials only, and outlier trials (more than three SDs away from the mean) were removed. A speed/accuracy trade-off score for emotion regulation conditions (i.e., where the "nogo" stimulus was an emotional expression) was computed by taking the ratio of z-transformed reaction time (normalized to the standard deviation of overall reaction time) to $\mathrm{z}$-transformed false alarm rate (normalized to the standard deviation of overall false alarm rate) for conditions when emotional faces were "nogo" stimuli. This ratio of reaction time to false alarm was the speed/accuracy trade-off score during emotion regulation conditions.

\section{RESULTS}

\section{D-PRIME}

A repeated measures ANOVA with between subjects factors of age group (children, adolescents, adults) and gender (male, female) and within subjects factors of emotion (happy, fear, angry, sad) and stimulus type (emotion as "go" or "nogo") was performed on the primary dependent measure of d-prime. There was a two-way interaction between stimulus type by emotion $[F(3,282)=6.18$, $\left.p<10^{-4}, \eta_{p}^{2}=0.06\right]$ and main effects of age group $[F(2,94)=43.17$, $\left.p<10^{-14}, \eta_{p}^{2}=0.48\right]$, gender $\left[F(1,94)=24.23, p<0.008, \eta_{p}^{2}=0.07\right]$, emotion $\left.F(3,282)=48.36, p<10^{-25}, \eta_{\mathrm{p}}^{2}=0.34\right)$, and stimulus type $\left[F(1,94)=32.89, p<10^{-7}, \eta_{\mathrm{p}}^{2}=0.26\right]$.

\section{Emotion effects}

As illustrated in Figure 2, post hoc t-tests showed that d-prime was significantly lower when happy $\left(t=6.43, p<10^{-9}\right)$ and sad faces $\left(t=3.90, p<10^{-4}\right)$ were the "nogo" stimuli compared to when they were the "go" stimuli, and across all subjects, there was a main effect for stimulus type such that d-prime was lower for those conditions when emotional faces were "nogo" stimuli $[$ mean $(\mathrm{SD})=2.10$ (0.92)] compared to when neutral faces were "nogo" stimuli [mean $\left.(\mathrm{SD})=2.46(0.96) ; t=5.89, p<10^{-8}\right]$. Across all subjects, discrimination was better for fear [mean $(\mathrm{SD})=2.61(1.16) ; t$ (relative to angry) $=6.79, p<10^{-10} ; t$ (relative to sad $\left.)=8.47, p<10^{-13}\right]$ and happy $[$ mean $(\mathrm{SD})=2.70(0.99) ; t$ (relative to angry $)=8.82$, $p<10^{-14} ; t$ (relative to sad) $=10.86, p<10^{-18}$, which did not differ from each other $(t=0.91, \mathrm{~ns})$, and worse for angry [mean $(\mathrm{SD})=2.00(0.92)]$, and sad [mean $(\mathrm{SD})=1.82(1.01)]$, which had the worst discrimination rate $[$ (relative to angry) $=2.479$, $p<0.015$ ] regardless of age.

\section{Gender effects}

D-prime was overall higher for females [mean (SD) $=2.42(0.95)$ ] who had more accurate emotion recognition than males [mean $(\mathrm{SD})=2.14(0.80)]$.

\section{Developmental effects}

Discrimination increased with age, such that children had the lowest d-prime scores [mean $(\mathrm{SD})=1.74(0.73) ; t$ (relative to adolescents $)=5.08, p<10^{-6} ; t$ (relative to adults $\left.)=8.52, p<10^{-12}\right]$, followed by adolescents [mean $(\mathrm{SD})=2.64(0.68) ; t$ (relative to adults $)=2.97, p<0.005$ ] and then adults [mean $(\mathrm{SD})=3.14$ $(0.50)]$. There was no significant quadratic effect across age groups in d-prime for emotional $(p=0.17)$ or neutral $(p=0.42)$ "go" conditions.

\section{FALSE ALARMS}

A repeated measures ANOVA with between subjects factors of age group (children, adolescents, adults) and gender (male, female) and within subjects factors of emotion (happy, fear, angry, sad) and stimulus type (emotion as "go" or "nogo") was performed on the dependent measure of false alarm rate. As shown in Figure 3, there were two-way interactions of age by stimulus type $\left[F(2,94)=5.88, p<0.04, \eta_{\mathrm{p}}^{2}=0.27\right]$ and emotion by stimulus type $\left[F(3,282)=3.67, p<0.015, \eta_{p}^{2}=0.04\right]$, and there were main effects of age $\left[F(2,94)=40.29, p<10^{-13}, \eta_{p}^{2}=0.46\right]$, emotion $\left[F(3,282)=31.21, p<10^{-17}, \eta_{\mathrm{p}}^{2}=0.25\right]$, and stimulus type $\left[F(1,94)=34.18, p<10^{-8}, \eta_{\mathrm{p}}^{2}=0.27\right]$.

\section{Emotion effects}

Regardless of age, emotion interacted with stimulus type such that false alarm rate was always higher when the emotional face was the "nogo" stimulus but this difference was smallest for fear, where both conditions that included fear faces had a high false alarm rate [mean (SD): fear as "go" $=0.18(0.22)$; fear as "nogo" $=0.22(0.23)]$. Subjects 


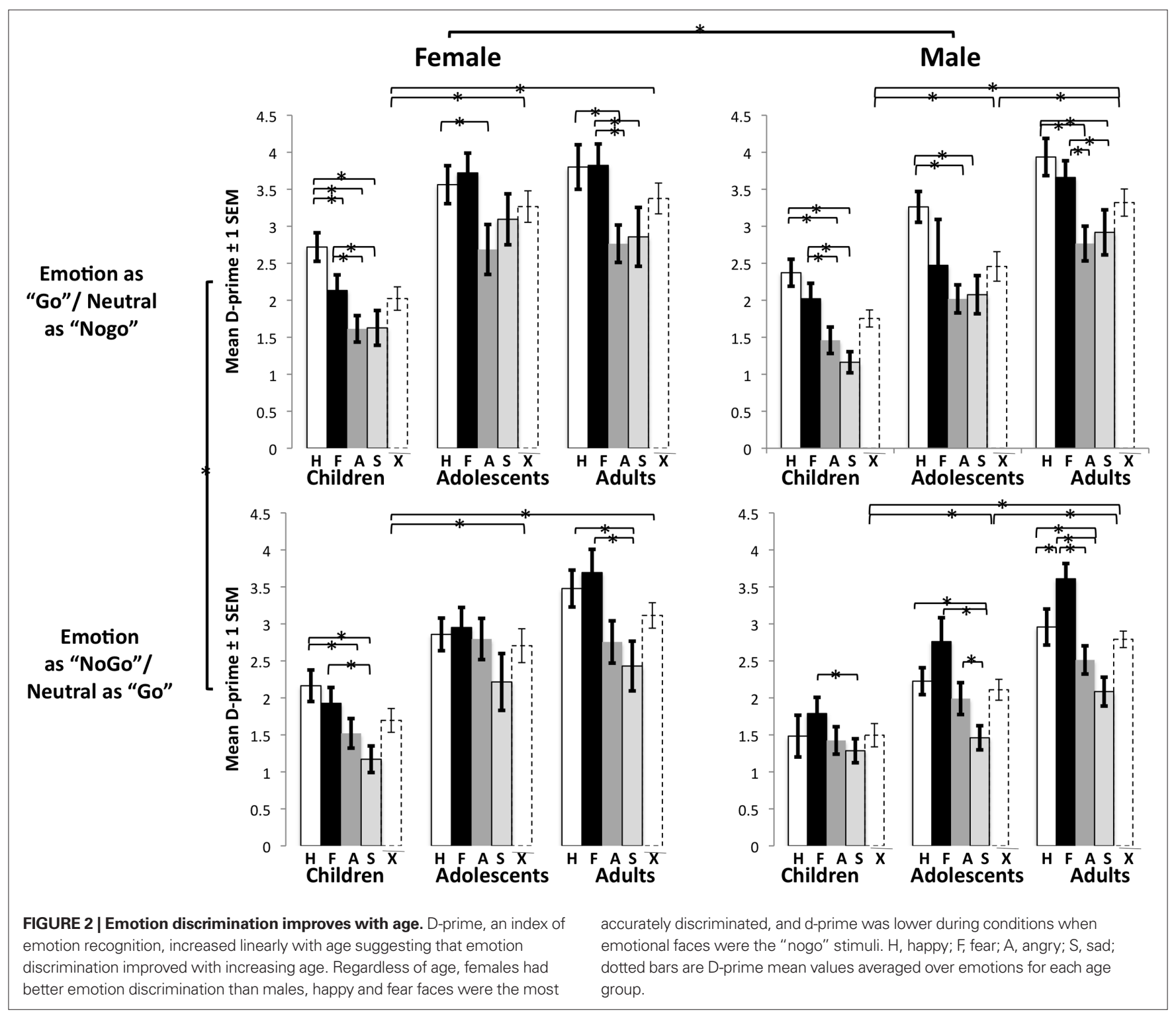

had a higher false alarm rate when any emotional face was the "nogo" stimulus [mean $(\mathrm{SD})=0.30(0.19)]$ compared to when an emotional face was the "go" stimulus [mean $(\mathrm{SD})=0.21(0.17) ; t=7.43$, $\left.p<10^{-11}\right)$. Across stimulus types, the greatest false alarm rate was for sad $\left[\right.$ mean $(\mathrm{SD})=0.34(0.21) ; t$ (relative to angry) $=5.16, p<10^{-6}$; $t($ relative to fear $)=8.76, p<10^{-14} ; t$ (relative to happy) $=10.07$, $p<10^{-17}$, followed by angry [mean $(\mathrm{SD})=0.27(0.19) ; t$ (relative to fear $)=3.68, p<10^{-4} ; t$ (relative to happy) $\left.=5.28, p<10^{-7}\right]$, and then fear $[$ mean $(S D)=0.20(0.21)]$ and happy $[$ mean $(S D)=0.19$ (0.16)], which did not differ from each other $(t=0.77$, ns).

\section{Developmental effects}

Post hoc tests showed that false alarm rate was higher for children $\left[\right.$ mean $(S D)=0.36(0.16) ; t($ relative to adolescents $)=5.43, p<10^{-7}$; $t$ (relative to adults $)=7.47, p<10^{-10}$ ] than it was for adolescents $[$ mean $(\mathrm{SD})=0.17(0.09) ; t$ (relative to adults $)=3.08, p<0.004]$, who had a higher false alarm rate than adults $[$ mean $(S D)=0.10$
(0.06)]. As can be seen by the age group $\times$ stimulus type interaction in Figure 3, emotion regulation (that is, inhibiting a prepotent behavioral response in the context of emotional information) showed greater age-group related improvement relative to cognitive control (that is, inhibiting a prepotent behavioral response in the context of neutral information). The stimulus type by age interaction was significant, such that false alarm rate to emotional "nogo" stimuli was largest relative to neutral "nogo" stimuli for children [mean (SD): emotional "nogo" $=0.42(0.17)$; neutral "nogo" $=0.29$ (0.17); $t=6.92, p<10^{-9}$ ], followed by adolescents [mean (SD): emotional "nogo" $=0.19(0.11)$; neural "nogo" $=0.14(0.09) ; t=2.53$, $p<0.02$ ], and then adults [mean (SD): emotional "nogo" $=0.12$ (0.08); neutral "nogo" $=0.08(0.07) ; t=2.69, p<0.015]$. We tested for the presence of a quadratic trend for age (separately for "nogo" conditions that were emotional and neutral), which was significant only for emotional "nogo" trials $(p<0.05)$ but not for neutral "nogo" trials $(p=0.18)$. 


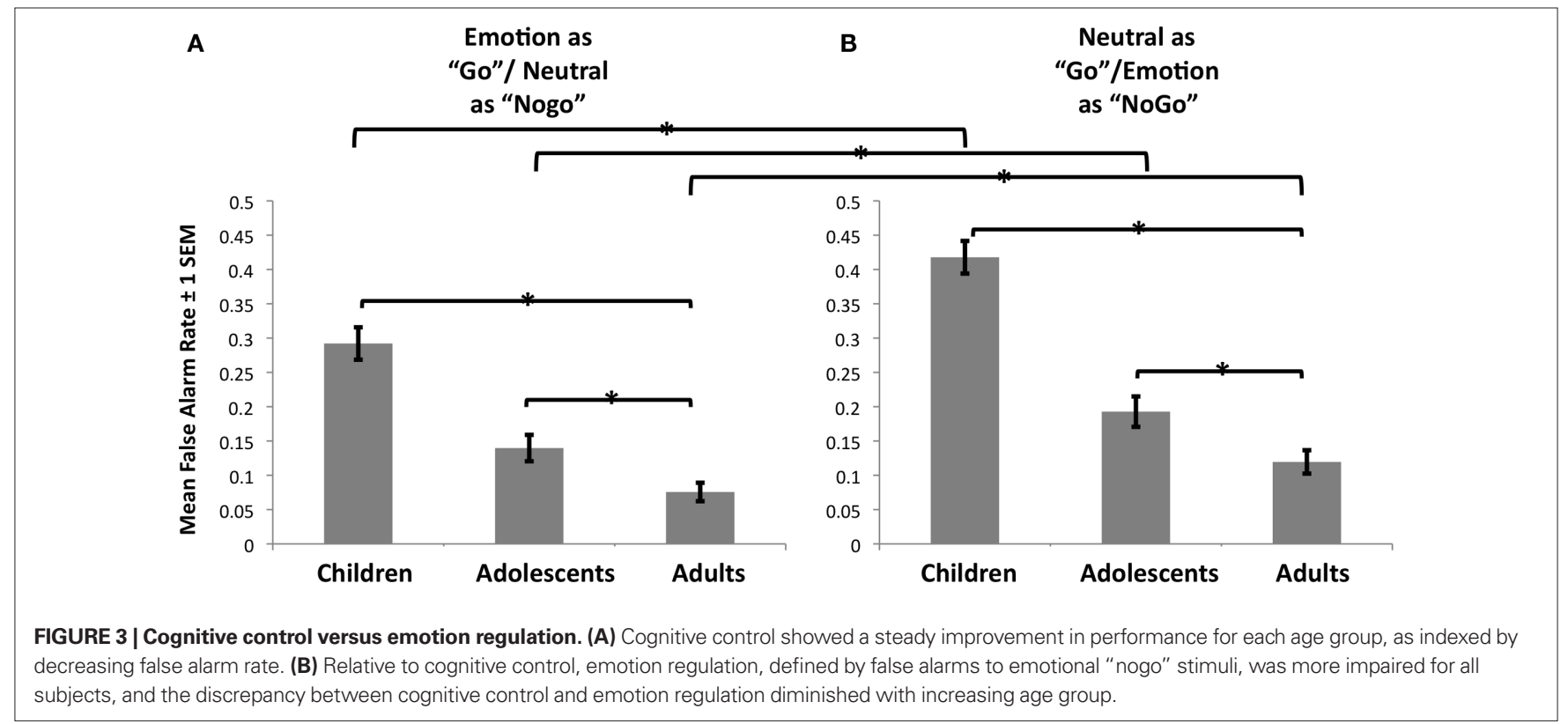

\section{MISS RATE}

A repeated measures ANOVA with between subjects factors of age group (children, adolescents, adults) and gender (male, female) and within subjects factors of emotion (happy, fear, angry, sad) and stimulus type (emotion as "go" or "nogo") was performed on the dependent measure of miss rate. There was a two-way interaction of emotion by stimulus type $\left[F(3,282)=6.17, p<10^{-4}, \eta_{p}^{2}=0.06\right]$ and an overall main effect of emotion $\left[F(3,282)=7.99, p<10^{-5}, \eta_{p}^{2}=0.08\right]$.

\section{Emotion effects}

Regardless of age, miss rate was especially low when happy faces were the "go" stimulus [mean $(\mathrm{SD})=0.08(0.07)]$ relative to when it was the "nogo" stimulus [mean $(\mathrm{SD})=0.15(0.18) ; t=3.97$, $p<10^{-4}$ and especially high when sad was the "go" stimulus $[$ mean $(\mathrm{SD})=0.18(0.17)]$ compared to when it was the "nogo" stimulus [mean $(\mathrm{SD})=0.14(0.15) ; t=2.03, p<0.05]$, suggesting that subjects are accurate in positive discrimination (pressing for the "go" stimulus) of happy faces and significantly inaccurate at positively discriminating sad faces. Overall, happy [mean $(\mathrm{SD})=0.11(0.10) ; t$ (relative to fear $)=1.55, \mathrm{~ns}, t$ (relative to angry) $\left.=4.93, p<10^{-6}\right]$ and fear $[$ mean $(\mathrm{SD})=0.13(0.13) ; t$ $($ relative to angry $)=2.84, p<0.005 ; t$ (relative to sad $)=2.48$, $p<0.015$ ] had the lowest miss rates, and angry [mean $(\mathrm{SD})=0.16$ $(0.11) ; t$ (relative to sad $)=0.19, \mathrm{~ns}]$ and sad $[$ mean $(\mathrm{SD})=0.16$ (0.13)] had the highest miss rates. We tested for the presence of a quadratic trend for age (separately for "go" conditions that were emotional and neutral), which was not significant for either, $p=0.46$ and 0.58 , respectively.

\section{REACTION TIME}

A repeated measures ANOVA with between subjects factors of age group (children, adolescents, adults) and gender (male, female) and within subjects factors of emotion (happy, fear, angry, sad) and stimulus type (emotion as "go" or "nogo") was performed on the dependent measure of reaction time. There was a two-way interaction of emotion by stimulus type $\left[F(3,282)=10.96, p<10^{-7}\right.$, $\left.\eta_{p}^{2}=0.02\right]$, and an overall main effect of emotion $[F(3,282)=22.79$, $\left.10^{-13}, \eta_{\mathrm{p}}^{2}=0.20\right]$.

\section{Emotion effects}

Regardless of age, reaction times were faster for happy faces when they were the "go" stimulus [mean $(\mathrm{SD})=460 \mathrm{~ms}(83)]$ compared to when they were the "nogo" stimulus [mean $(\mathrm{SD})=493 \mathrm{~ms}(88)$; $\left.t=4.72, p<10^{-6}\right]$ and slower when sad faces were the "go" stimulus $[$ mean $(\mathrm{SD})=528 \mathrm{~ms}(81)]$ compared to when they were the "nogo" stimulus [mean $(\mathrm{SD})=511 \mathrm{~ms}(105) ; t=2.10, p<0.05]$. Overall, subjects were fastest at discriminating happy $[$ mean $(\mathrm{SD})=476 \mathrm{~ms}$ (78); $t$ (relative to fear $)=3.57, p<0.001 ; t$ (relative to angry) $=4.86$, $p<10^{-6} ; t$ (relative to sad) $=7.65, p<10^{-11}$, followed by fear [mean $(\mathrm{SD})=497 \mathrm{~ms}(93)]$ and angry faces $[$ mean $(\mathrm{SD})=501 \mathrm{~ms}(82)]$, which did not differ from each other $(t=0.82$, ns), and slowest to sad faces $[$ mean $(\mathrm{SD})=520 \mathrm{~ms}(85) ; t$ (relative to fear $)=4.02$, $p<10^{-4} ; t$ (relative to angry) $=4.14, p<10^{-5}$ ]. We tested for the presence of a quadratic trend for age (separately for "go" conditions that were emotional and neutral), which was significant for emotion as "go" trials only, $p<0.015$ and not for neutral as "go" trials $(p=0.07)$, suggesting that adolescents are the fastest group for the emotional "go" trials, but not for neutral "go" trials.

\section{SPEED/ACCURACY TRADE-OFF}

An ANOVA with between subject factors of age group (children, adolescents, and adults) and gender (male, female) was performed on the dependent measure of speed/accuracy trade-off. There was an interaction with age and gender $[F(2,98)=3.15, p<0.05$, $\left.\eta_{\mathrm{p}}^{2}=0.06\right]$.

\section{Age by gender interaction}

Post hoc $t$-tests showed that the source of the interaction was due to adolescent males' higher speed/accuracy trade-off relative to children $(t=2.70, p<0.015)$ and adults $(t=2.38, p<0.03)$, who 
did not differ from each other (Figure $4 ; t=1.23$, ns). No other comparisons were significant. We tested for the presence of a quadratic trend for age (separately for each gender because age interacted with gender), which was not significant for female subjects, $p=0.68$, but was significant for male subjects for an inverted-U shaped curve, $p<0.008$, confirming the results from the ANOVA as shown in Figure 4.

\section{DISCUSSION}

Emotional and cognitive processes interact to influence emotion regulation, such that mature emotion regulation involves the ability to not only recognize the emotional significance of perceived stimuli, but to regulate behavior in the context of emotional information. We found support for the hypothesis that (1) emotional information can impair cognitive control processes, and (2) that there would be unique age-group related improvements in the skills of cognitive control, emotion regulation, and emotion discrimination. We summarize the findings here and discuss them each in more detail below. We provided evidence that emotional information can interfere with cognitive control by showing higher false alarm rates in the context of emotional relative to neutral "nogo" stimuli. A similar result was found in a recent study using a "day/ night" task with iconic drawings of happy and sad faces (Lagattuta et al., in press), and our results showed that photographs of faces, expressing various emotions, create a similar performance deficit. Furthermore, each age group showed unique patterns of emotion discrimination, cognitive control, and emotion regulation skills, at least as measured in a cross-sectional sample. Cognitive control improved with increasing age group in a linear fashion. An age group by stimulus type interaction showed that while false alarm rate to emotional "nogo" stimuli was higher than it was to neutral faces for all groups, the cost to false alarm rate caused by emotional information decreased with increasing age. By traditional ANOVA, reaction time did not change across the three age groups, but there was a significant quadratic trend for emotional "go" stimuli, suggesting that adolescents were responding faster than the other age groups to the emotional stimuli. Importantly, the adolescent group did not show this rapid response time for neutral "go" stimuli (when emotional faces were the "nogo" stimuli). Consistent with these reaction time data, there was an inverted-U shaped curve for speed/accuracy trade-off for conditions when "nogo" stimuli were emotional in the male subjects, suggesting that despite adolescent males responding fast to emotional "go" stimuli, they showed a significant trade-off between speed and accuracy when inhibiting pressing to emotional "nogo" stimuli. Consistent with previous literature (McClure, 2000; Herba and Phillips, 2004; Herba et al., 2006; Thomas et al., 2007), we showed that emotion discrimination (d-prime) was poorest for children, but in light of the miss rate data, which showed children performing at the same level as adults, these d-prime data suggest that emotion discrimination is most impaired when negatively discriminating an emotional expression (i.e., withholding a press for the emotional "nogo" stimulus). Thus, there are certain conditions under which children's ability to make simple emotional discriminations based on facial information is more impaired than in others. When making positive discriminations (pressing for the emotional "go" face), child performance was at par with the adult group.

\section{EMOTION RECOGNITION}

Regardless of age, certain emotions are more readily discriminated than others. The emotions of fear and happy were recognized as more distinct from neutral faces as indexed by higher d-prime values and fast reaction times for all age groups, and sad was recognized most poorly, with low accuracy and longest response times. It is worth noting that although this task involved a simple emotion discrimination task (discriminating an emotional expression from neutral), the poor performance for sad faces has been noted elsewhere (Herba et al., 2006) and may be in part related to the greater perceptual similarity between sad and neutral faces. Across emotions, there were gender and age differences in emotion discrimination. Female subjects were overall more accurate than males in discriminating emotions as indexed by higher d-prime

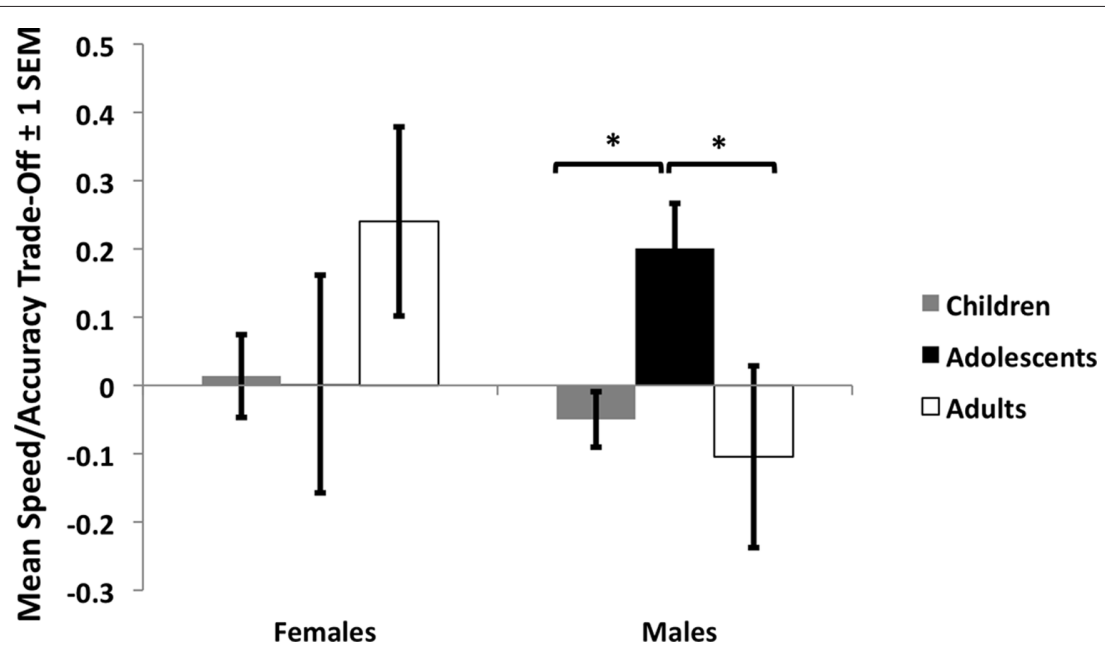

FIGURE 4 | Speed/accuracy trade-off during adolescence. Adolescent males showed a high speed/accuracy trade-off during conditions when emotional faces were the "nogo" stimuli, such that relative to children and adults, adolescents slowed down response time to regulate their responding during these conditions. 
values, consistent with previous reports of females' superior ability in facial expression processing (McClure, 2000). Children were less accurate at emotion recognition than adolescents who were less accurate than adults, and the linear age-group related change in d-prime suggests that the ability to discriminate emotions gradually, but consistently improves with age, a replication of previous finding showing age-related changes in emotion discrimination across these ages (Lenti et al., 1999; McClure, 2000; Herba and Phillips, 2004; Herba et al., 2006; Thomas et al., 2007), where improvements in facial expression perception are observed into early adulthood. The miss rate (i.e., 1-hit rate) indexes the likelihood that a subject does not press for a target expression and provides a very important confirmation of whether or not subjects could positively discriminate a named expression. The miss rate was low (i.e., the hit rate was high) and did not differ for the three age groups, suggesting that all subjects were able to positively discriminate (via button presses) each named expression. The lack of age-group related change in miss rates despite age-group related change in d-prime scores suggests that children are able to positively discriminate emotions quite early, but that children were impaired when their emotion discrimination relied on inhibiting a response (correctly inhibiting "nogo" responding). It is important to note that the task used in this manuscript requires subjects to positively identify given expressions, but we cannot determine from this task whether or not subjects fully understood the emotional meaning of each expression. Another limitation of this task is that we rely on facial expressions, and often standard facial expression sets include only one positive expression (happy), although we present multiple models posing this expression. Future studies that employ the Emotional Go/Nogo task may benefit from including multiple positive expressions (e.g., exuberant) to more fully explore the processing of positive expressions.

\section{COGNITIVE CONTROL}

False alarm rate was used to index cognitive control. There was a significant age-group related decrease in false alarm rate from childhood to adolescence and from adolescence to adulthood suggesting a linear improvement in cognitive control for each increasing age group. The age-related change in mean false alarm rate but not mean miss rate suggests that the age-group related change in performance was due to changes in cognitive control. This finding replicates findings from previous studies showing that cognitive control is a late developing process with improvement well into adulthood (Bunge et al., 2002; Durston et al., 2002; Somerville and Casey, 2010). The added value of the Emotional Go/Nogo task is in its ability to directly compare these changes in cognitive control to age-related changes in emotion regulation.

\section{EMOTION REGULATION}

Emotion regulation was defined in this study as the ability to regulate impulsive responses in the context of emotional information. Thus, false alarm rate during conditions when emotional faces were the "nogo" stimulus was used as our index of emotion regulation (i.e., maintaining cognitive control in the presence of emotional information). False alarm errors to emotional "nogo" stimuli did not differ by valence of expressions, suggesting that both approach-related information (e.g., happy) and avoid-related information (e.g., fear, angry) were equally effective in disrupting behavioral performance relative to neutral distractors. False alarm rate to emotional "nogo" stimuli was worse than to neutral "nogo" stimuli across all subjects, but performance was especially poor in children. Although emotional "nogo" stimuli resulted in a performance deficit across age groups, the deficit decreased with increasing age, suggesting that emotion regulation is a slower developing process than general cognitive control. Future longitudinal data is required to confirm this interpretation. A recent longitudinal study using a modified "day/night" task that included cartoon happy and sad facial expressions showed that emotional conditions resulted in worse inhibitory control performance than did the non-emotional conditions across development, consistent with the results from the current study. This previous experiment suggested that it was the interaction between emotion and the more "cool" aspects of executive function that resulted in the greater difficulty for emotional stimuli in general (Lagattuta et al., in press). In contrast to the current study, this study did not find that emotional conditions interacted with age group (i.e., there was no developmental change in the size of the performance deficit). This experimental difference may either suggest that there are no developmental changes in emotion regulation when studied longitudinally, or alternatively, that discriminating real-life images of facial expressions in the context of an inhibitory control task can reveal age-related improvements in emotion regulation. The Emotional Go/Nogo task is additionally useful in that it can be computerized for neuroimaging use to examine the neural correlates that underlie changes in emotion regulation. Moreover, it provides an index of emotion discrimination (with both hit and miss data) across multiple facial expressions.

The child group had a high error rate in conjunction with the slowest response time. While children also had the lowest d-prime scores for emotion discrimination (as described above) presenting the possibility that poor emotion discrimination increased false alarm errors, both the low miss rate in the child group and the fewer number of false alarms to neutral "nogo" stimuli decrease the likelihood that, in these data, emotion discrimination was a contributing factor to emotion regulation. Adolescents, in contrast, were the group that responded to the emotional "go" stimuli the fastest, as evidenced by the significant quadratic trend. However, there was no quadratic effect for those conditions when neutral faces were the "go" stimuli (when emotional faces were the "nogo" stimuli) suggesting that adolescents were faster when approaching emotional information. This speed to emotional stimuli is consistent with previous findings that adolescents tend to accelerate responding in emotional contexts (Steinberg et al., 2008). However, for male subjects, performance in the emotion regulation condition required a cost to relative response time in that adolescent subjects slowed down in the blocks where the "nogo" stimuli were emotional. In other words, to exhibit more mature behavioral performance in accuracy, adolescent males employed the potentially fragile strategy of slowing down response time when faced with emotional "nogo" stimuli. Children did not modify mean reaction time during emotional "nogo" trials and consequently made many false alarm errors during these conditions, and adults did not show this cost in reaction time, yet were able to demonstrate high accuracy. Therefore, adolescent males 
seem to be highly sensitive to the emotional information provided in the "nogo" stimuli which forces them to either slow down or make regulatory errors. This task also requires a second form of emotion regulation, which is the ability to regulate behavior while approaching potentially negative information (e.g., angry faces). However, the lack of an interaction between age group, emotion, and stimulus type suggests that this type of emotion regulation does not undergo age-related change that can be measured by this version of the task.

The improvement in false alarms to emotional information that adolescents seem to show, at least for males, can only occur when subjects increased reaction time suggesting that emotional information during adolescence has the potential to interrupt regulatory ability if the adolescent does not slow down response time. Overall, both male and female adolescents are the fastest group to approach emotional information. Thus, relative to adulthood, adolescence may be a period when individuals are at elevated risk for behavioral errors under emotional conditions. Our findings lend support for a dual-system model of emotion regulation (Casey et al., 2008; Steinberg et al., 2008), which posits that subcortical regions and associated emotion processing systems develop before (reaching a peak in adolescence) prefrontal cortical regions that support regulatory functions, making adolescent emotion regulation weaker than adults (Somerville and Casey, 2010). These distinct trajectories present an adolescent-limited imbalance in neural systems such that during adolescence, activity of emotional systems operate without fully mature regulatory control processes. Although our results

\section{REFERENCES}

Bunge, S. A., Dudukovic, N. M., Thomason, M. E., Vaidya, C. J., and Gabrieli, J. D. (2002). Immature frontal lobe contributions to cognitive control in children: evidence from fMRI. Neuron 33, 301-311.

Campos, J. J., Frankel, C. B., and Camras, L. (2004). On the nature of emotion regulation. Child Dev. 75, 377-394.

Casey, B. J., Getz, S., and Galvan, A. (2008). The adolescent brain. Dev. Rev. 28, 62-77.

Casey, B. J., Tottenham, N., and Fossella, J. (2002). Clinical, imaging, lesion, and genetic approaches toward a model of cognitive control. Dev. Psychobiol. 40, 237-254.

Casey, B. J., Trainor, R. J., Orendi, J. L., Schubert, A. B., Nystrom, L. E., Giedd, J. N., Castellanos, F. X., Haxby, J. V., Noll, D. C., Cohen, J. D., Forman, S. D., Dahl, R. E., and Rapoport, J. L. (1997). A Developmental functional MRI study of prefrontal activation during performance of a go-no-go task. J. Cogn. Neurosci. 9, 835-847.

Cohen, J. D., MacWhinney, B., Flatt, M., and Provost, J. (1993). PsyScope: a new graphic interactive environment for designing psychology experiments. Behav. Res. Methods Instrum. Comput. 25, 257-271.
Cole, P. M., Martin, S. E., and Dennis, T. A. (2004). Emotion regulation as a scientific construct: methodological challenges and directions for child development research. Child Dev. 75, 317-333.

Diamond, A. (1985). Development of the ability to use recall to guide action, as indicated by infants' performance on the A, not B. Child Dev. $56,868-883$.

Duncan, J. (2001). An adaptive coding model of neural function in prefrontal cortex. Nat. Rev. Neurosci. 2, 820-829.

Durston, S., Davidson, M. C., Tottenham, N., Galvan, A., Spicer, J., Fossella, J. A., and Casey, B. J. (2006). A shift from diffuse to focal cortical activity with development. Dev. Sci. 9, 1-8.

Durston, S., Thomas, K. M., Yang, Y., Ulug, A. M., Zimmerman, R. D., and Casey, B. J. (2002). A neural basis for the development of inhibitory control. Dev. Sci. 5, F9-F16.

Durston, S., Tottenham, N., Thomas, K. M., Davidson, M. C., Eigsti, I. M., Yang, Y., Ulug, A. M., and Casey, B. J. (2003). Differential patterns of striatal activation in young children with and without ADHD. Biol. Psychiatry 53, 871-878.

Eisenberg, N., Champion, C., and Ma, Y. (2004). Emotion-related regulation:

await confirmation from longitudinal designs, they are important behavioral data that add support for this neurobiological model of developmental change.

The ability to regulate behaviors in the context of emotionally distracting information is one of several aspects that contributes to emotion regulation, and others include the ability to make decisions based on emotional information and the ability to reappraise emotionally arousing information (Campos et al., 2004). Characterizing age-related performance is important in understanding how behaviors related to emotion regulation emerge cross development and allows for the identification of developmental windows when a child may be especially vulnerable to influences that can shape these behaviors (e.g., emotion regulation in adolescence). These findings provide normative behavioral data for the interpretations of future neuroimaging and clinical studies in developing populations. Obtaining a general index of cognitive control as well as emotion regulation in a single measure is a valuable tool not only for use in identifying atypical patterns of development like those identified in children with internalizing and externalizing behaviors (Durston et al., 2003; Tottenham et al., 2011), but also for future studies that examine the mechanisms of change in typical child and adolescent behaviors and how the processes that contribute to emotion regulation are constructed across developmental time.

\section{ACKNOWLEDGMENT}

Supported in part by R01 MH73175, R01 DA018879, and P50 MH 079513 (B.J. Casey).

an emerging construct. Merrill Palmer Q. 50, 236-259.

Ekman, P., and Friesen, W. V. (1976). Pictures of Facial Affect. Palo Alto, CA: Consulting Psychologists Press.

Flom, R., and Bahrick, L. E. (2007). The development of infant discrimination of affect in multimodal and unimodal stimulation: the role of intersensory redundancy. Dev. Psychol.43, 238-252.

Gross, J. J. (1998). Antecedent- and response-focused emotion regulation: divergent consequences for experience, expression, and physiology. J. Pers. Soc. Psychol. 74, 224-237.

Hare, T. A., Tottenham, N., Galvan, A., Voss, H. U., Glover, G. H., and Casey, B. J. (2008). Biological substrates of emotional reactivity and regulation in adolescence during an emotional gonogo task. Biol. Psychiatry 63, 927-934.

Herba, C., and Phillips, M. (2004). Annotation: development of facial expression recognition from childhood to adolescence: behavioural and neurological perspectives. J. Child Psychol. Psychiatry 45, 1185-1198.

Herba, C. M., Landau, S., Russell, T., Ecker, C., and Phillips, M. L. (2006). The development of emotion-processing in children: effects of age, emotion, and intensity. J. Child Psychol. Psychiatry 47, 1098-1106.
Kindt, M., Bierman, D., and Brosschot, J. F. (1997). Cognitive bias in spider fear and control children: assessment of emotional interference by a card format and a single-trial format of the Stroop task. J. Exp. Child Psychol. 66, 163-179.

Kochanska, G., Murray, K. T., and Harlan, E. T. (2000). Effortful control in early childhood: continuity and change, antecedents, and implications for social development. Dev. Psychol.36,220-232.

Lagattuta, C.H., Sayfan, L., and Monsour, M. (in press). A new measure for assessing executive function across a wide age range: children and adults find happy-sad more difficult than day-night. Dev. Sci.

Lenti, C., Lenti-Boero, D., and Giacobbe, A. (1999). Decoding of emotional expressions in children and adolescents. Percept. Mot. Skills 89, 808-814.

Marsh, R., Zhu, H., Schultz, R. T., Quackenbush, G., Royal, J., Skudlarski, P., and Peterson, B. S. (2006). A developmental fMRI study of self-regulatory control. Hum. Brain Mapp. 27,848-863.

McClure, E. B. (2000). A meta-analytic review of sex differences in facial expression processing and their development in infants, children, and adolescents. Psychol. Bull. 126, 424-453.

Miller, E. K., and Cohen, J. D. (2001). An integrative theory of prefrontal cor- 
tex function. Annu. Rev. Neurosci. 24, 167-202.

Mischel, W., and Underwood, B. (1974). Instrumental ideation in delay of gratification. Child Dev. 45, 1083-1088.

Munakata, Y., and Yerys, B. E. (2001). All together now: when dissociations between knowledge and action disappear. Psychol. Sci. 12, 335-337.

Nelson, C. A., and Dolgin, K. G. (1985). The generalized discrimination of facial expressions by seven-month-old infants. Child Dev. 56, 58-61.

Ochsner, K. N., and Gross, J. J. (2005). The cognitive control of emotion. Trends Cogn. Sci. 9, 242-249.

Oosterlaan, J., Logan, G. D., and Sergeant, J. A. (1998). Response inhibition in $\mathrm{AD} / \mathrm{HD}, \mathrm{CD}$, comorbid AD/HD + CD, anxious, and control children: a metaanalysis of studies with the stop task. J. Child Psychol. Psychiatry 39, 411-425.
Piaget, J. (1954). The Construction of Reality in the Child. New York: Basic Books.

Somerville, L. H., and Casey, B. J. (2010). Developmental neurobiology of cognitive control and motivational systems. Curr. Opin. Neurobiol. 20, 236-241.

Somerville, L. H., Hare, T. A., and Casey, B. J. (in press). Frontostriatal maturation predicts cognitive control failure to appetitive cues in adolescents. J. Cogn. Neurosci.doi: 10.1162/jocn.2010.21572. [Epub ahead of print].

Steinberg, L., Albert, D., Cauffman, E. Banich, M., Graham, S., and Woolard, J. (2008). Age differences in sensation seeking and impulsivity as indexed by behavior and self-report: evidence for a dual systems model. Dev. Psychol. 44, 1764-1778.

Thomas, L.A., DeBellis, M.D., Graham, R., and LaBar, K. S. (2007). Development of emotional facial recognition in late childhood and adolescence. Dev. Sci. 10, 547-558.

Tipper, S. P., Bourque, T. A., Anderson, S. H., and Brehaut, J. C. (1989). Mechanisms of attention: a developmental study. J. Exp. Child Psychol. 48, 353-378.

Tottenham, N., Hare, T. A., Millner, A., Gilhooly, T., Zevin, J. D., and Casey, B. J. (2011). Elevated amygdala response to faces following early deprivation. Dev. Sci. 14, 190-204.

Wechsler, D. (1999). The Wechsler Abbreviated Scale of Intelligence. New York: The Psychological Corporation. Zelazo, P. D., Frye, D., and Rapus, T. (1996). An age-related dissociation between knowing rules and using them. Cogn. Dev. 11, 37-63.

Conflict of Interest Statement: The authors declare that the research was con- ducted in the absence of any commercial or financial relationships that could be construed as a potential conflict of interest.

Received: 17 July 2010; accepted: 24 February 2011; published online: 16 March 2011.

Citation: Tottenham N, Hare TA and Casey BJ (2011) Behavioral assessment of emotion discrimination, emotion regulation, and cognitive control in childhood, adolescence, and adulthood. Front. Psychology 2:39. doi: 10.3389/fpsyg.2011.00039

This article was submitted to Frontiers in Developmental Psychology, a specialty of Frontiers in Psychology.

Copyright (C) 2011 Tottenham, Hare and Casey. This is an open-access article subject to an exclusive license agreement between the authors and Frontiers Media SA, which permits unrestricted use, distribution, and reproduction in any medium, provided the original authors and source are credited. 